\title{
Comparación del impacto de libros y artículos de investigadores españoles de comunicación a través de Google Scholar en 2019
}

\author{
Javier Marzal-Felici*, Aarón Rodríguez-Serrano**, María Soler-Campillo*** \\ Universitat Jaume I, Castellón de la Plana. \\ * Correo-e: marzal@uji.es | ORCID iD: http://orcid.org/0000-0002-2462-1122 \\ ** Correo-e: serranoa@uji.es | ORCID iD: http://orcid.org/0000-0002-3858-1045 \\ *** Correo-e: soler@uji.es | ORCID iD: http://orcid.org/0000-0002-3237-8038
}

Recibido: 20-12-19; 2a versión: 01-04-20; Aceptado: 16-04-20; Publicado: 02-03-21

Resumen: En este artículo se propone un análisis del impacto de la investigación en comunicación a través del estudio comparado de la citación en los principales formatos de publicación -libros y artículos- en las áreas de periodismo y de comunicación audiovisual y publicidad. Para ello, se han tomado como referencia las tres publicaciones de mayor impacto de 281 investigadores de universidades públicas españolas, contando así con 843 trabajos que han arrojado un total de 72.993 citas según Google Scholar. La investigación concluye la convivencia armónica de formatos en este campo, si bien los libros muestran un mayor recorrido temporal mientras que las revistas son claramente elegidas por los investigadores de menor edad. Dichas conclusiones son finalmente contextualizadas en el marco de las actuales políticas de la investigación en España.

Palabras clave: impacto de la investigación; investigación en comunicación; bibliometría; Google Scholar; citación; artículo científico; libro académico; ANECA

\section{Comparison of the impact of books and articles by Spanish communication researchers through Google Scholar in 2019}

\begin{abstract}
This paper offers an analysis of the impact of research in communication through a comparative study of citation in main publication formats -books and papers- in the areas of journalism, audiovisual communication and advertising. With this aim, the three publications with greatest impact of 281 researchers ( 199 headlines and 82 professors) from Spanish public universities have been taken as a reference, thus counting 843 papers, that have yielded a total of 72,993 citations, according to Google Scholar. The research concludes that there is and harmonious coexistence of formats in this field, although the books show a longer time course while the journals are clearly chosen by the younger researchers. These conclusions are finally contextualized within the framework of current research policies in Spain.
\end{abstract}

Keywords: research impact; research in communication; bibliometry; Google Scholar; citation; scientific paper; academic book; ANECA.

Cómo citar este artículo/Citation: Marzal-Felici, J.; Rodríguez-Serrano, A., Soler-Campillo, M (2021). Comparación del impacto de libros y artículos de investigadores españoles de comunicación a través de Google Scholar en 2019. Revista Española de Documentación Científica, 44 (1), e288. https://doi.org/ 10.3989/redc.2021.1.1744

Copyright: (c) 2021 CSIC. Este es un artículo de acceso abierto bajo los términos de la licencia de uso y distribución Creative Commons Reconocimiento 4.0 Internacional (CC BY 4.0). 


\section{INTRODUCCIÓN}

Como ha sido destacado por diferentes estudiosos de la investigación en comunicación, en los últimos años se detecta, con claridad, un cambio epistemológico en la investigación en comunicación, que se concreta en unos rasgos característicos como el uso instrumental de la teoría, la adopción de un canon investigador dominante, de vocación marcadamente internacional, y un auge muy notable de la investigación cuantitativa y empírica (Saperas, 2016: Carrasco-Campos y Saperas-Lapiedra, 2016), en cuyo desarrollo y evolución ha tenido muy escasa influencia la producción científica procedente -por ser casi inexistente- de las universidades privadas (Carraco-Campos y otros, 2018). Los últimos años están marcados por un tendencia clara hacia una paulatina consolidación de instituciones como las revistas científicas españolas y de diferentes sociedades científicas (por citar unas pocas, cabe destacar la Asociación Española de Investigación de la Comunicación AEIC-, la Sociedad Española de Periodística -SEP-, la Unión Latina de Economía Política de la Información, Comunicación y Cultura -ULEPICC-, entre otras), cuyo reto más relevante es la internacionalización de la producción científica y la visibilización del campo científico, más allá de nuestras fronteras (Saperas, 2016).

El Informe Estrategia española de Ciencias y Tecnología y de Innovación 2013-2020, publicado por el Ministerio de Economía y Competitividad en 2012, proponía como uno de sus seis ejes prioritarios "el apoyo a la Internacionalización y promoción del Liderazgo Internacional del Sistema Español de Ciencia, Tecnología e Innovación, ya que representan un claro factor de competitividad y diferenciación que es imprescindible potenciar" (Ministerio de Economía y competitividad, 2010: 6). Una de las claves que explica el desarrollo exponencial del artículo como formato de publicación científica en los últimos años es, precisamente, la necesidad de cumplir con la internacionalización de la producción científica española, para así proyectarla hacia el exterior, y ganar de este modo en visibilidad y competitividad. El citado Informe establecía como objetivos concretos incrementar durante el periodo 2013-2020 en un 50\%, el porcentaje de publicaciones en revistas que se encuentran entre el $10 \%$ de las más citadas sobre el total de las publicaciones científicas del periodo, así como incrementar en un $10 \%$ el porcentaje de las publicaciones generadas por proyectos financiados con recursos públicos que se encuentren entre el $5 \%$ de los documentos más citados de su área a nivel mundial.
En ciertos aspectos, podríamos aducir que el punto de partida del citado Informe era un balance muy crítico de la producción científica española -probablemente, cargado de buenas razones e intenciones-, al señalar que en 2010 apenas el $2,99 \%$ de las publicaciones científicas del mundo eran españolas (desde un $2,28 \%$ en 2000 ), mientras que el gasto interno total en actividades de I+D en el periodo 2000-2011 se había multiplicado por tres (pasando de 5.719 a 14.184 millones de euros), y el personal empleado en actividades de I+D había pasado entre 2000-2011 de 120.618 a 215.079 en total (de 76.670 a 130.235 investigadores), es decir, se había multiplicado por dos. De este modo, el Informe revela que la productividad científica española alcanzó en 2010 sus valores más bajos, a pesar de haber triplicado su financiación y duplicado sus recursos humanos. El Informe también reconoce que España es uno de los países desarrollados que menos invierte en $I+D$ en relación con su PIB, en torno a 1,40\% en 2010 (en 2018, la inversión en I+D ha sido de 14.588 millones, el 1,20\% del PIB español).

Es indudable que el artículo científico constituye un formato de publicación que presenta grandes ventajas, como ha sido puesto de manifiesto por numerosos autores (Abadal, 2017; Matínez-Nicolas y otros, 2019). Entre sus principales características se puede destacar que se trata de un tipo de publicación de extensión reducida -en el campo de la comunicación suele oscilar entre 4.500 y 10.000 palabras, dependiendo de las disciplinas-, lo que facilita su escritura $y_{\text {, }}$ también, su lectura; su circulación por internet y, en especial, por las redes sociales académicas. Multiplica enormemente la difusión de resultados de investigación, los procesos editoriales de los artículos para revistas académicas suelen ser sensiblemente más rápidos que la edición de libros individuales o colectivos y, en consecuencia, sus procesos editoriales son menos costosos que los de la edición del libro (al menos de manera general, en especial con la edición digital, que el libro tiene más limitada, por el momento). La existencia de bases de datos bibliográficas muy relevantes que recogen la actividad de revistas y editoriales, principalmente Web of Science, contribuye, además, a mejorar notablemente la reputación y la marca de las instituciones, universidades o empresas que las publican (Repiso y otros, 2019). Otro aspecto muy relevante que diferencia la edición de revistas frente a la de libros es que en el primer caso la evaluación por pares es generalizada, mientras que en la edición del libro académico este modelo de evaluación es poco frecuente. 
No obstante, llama la atención otro aspecto del Informe del Ministerio de Economía y Competitividad de 2012 que no puede pasar desapercibido: en ningún momento se contempla el establecimiento de diferencias entre ámbitos científicos -básicamente entre las humanidades y ciencias sociales frente a las ciencias naturales y experimentales-. En la práctica, esto corre el riesgo de entenderse como un modo de uniformizar el pensamiento científico. Este último asunto no es en absoluto menor, puesto que la manera de difundir, divulgar y compartir la ciencia también condiciona, de manera muy relevante, la forma de investigar, esto es, de definir, estructurar, argumentar y articular el pensamiento científico. Parece sensato señalar que más allá del método hipotético-deductivo siguen existiendo multitud de aproximaciones de gran rigor e interés metodológico, así como una pluralidad de objetos de estudio susceptibles de configurar grandes retos en el diseño contemporáneo de investigaciones en comunicación. Las visiones más críticas del campo sostienen que nos encontramos ante:

Sistemas que establecen como prioridad cuantificar las cosas y que, a riesgo de simplificarlas, permiten traducirlas a datos. Podrán así viajar más rápido y ordenarse más fácilmente, empujando fuera de su lógica aquellos aspectos del pensamiento más complejos, ambiguos, matizados e incluso contradictorios (Zafra, 2017: 76).

Nuestra investigación propone el estudio de las publicaciones que pueden ser consideradas casos de éxito en el campo concreto de la citación desde la perspectiva concreta y exclusiva de las citas recibidas. Para ello, hemos acotado exclusivamente a profesores funcionarios (catedráticos y titulares) de las universidades públicas españolas. Peor o mejor, algo dicen las citas académicas -y algo cuentan, a juzgar por los últimos criterios disponibles por ANECA (aprobados en 2017) en los que se valora el elevado índice $\mathrm{h}$ del investigador/a como mérito complementario-. No es nuestro objetivo juzgar aquí dicho sistema -que ya ha sido topografiado con bastante precisión (Gorraiz, 2018)-, del mismo modo que no pretendemos entrar en la valoración cualitativa de los formatos de investigación.

Es en este contexto en el que queremos preguntarnos por el rendimiento de libros -tanto monográficos como capítulos en obras coordinadas- y revistas. Ciertamente, algunos trabajos precedentes ya han profundizado en las diferentes fortalezas y debilidades de cada formato (Soriano, 2008, 2017), si bien también ha quedado apuntado que "la evidencia empírica [de dichas brechas] es aún algo endeble" (Martínez-Nicolás y Carrasco-Campos, 2018). La presente investigación pretende, específicamente, dotar -aunque sea parcialmente- de esa "evidencia empírica" que permita ulteriores desarrollos críticos sobre la valoración de los currículums académicos e investigadores. Tanto las revistas académicas (Repiso y otros, 2019) como los libros (Giménez, 2016; Català, 2018; Zunzunegui, Zumalde, 2018) han contado y cuentan con buenas razones para defender su relevancia, su función social e investigadora, y en definitiva, su impacto.

Nosotros pretendemos, modestamente, arrojar algo de luz sobre los modos en los que la convivencia de ambos formatos en la escena académica española, y más concretamente, en el campo de la comunicación, ha propiciado casos de éxito en las últimas décadas. Para ello, el primer paso consistió en sistematizar la población de profesores funcionarios susceptibles de ser estudiada bibliométricamente en el campo de la comunicación de la universidad pública española. Gracias a ello, se pudieron trazar los siguientes objetivos:

1. Realizar un análisis individual de los tres casos con mayor citación bibliográfica de cada investigador para poder determinar el alcance de los diferentes canales de publicación.

2. Realizar un análisis global de los formatos de citación por categorías profesionales y área de conocimiento (comunicación audiovisual y publicidad / periodismo).

3. Señalar aquellas diferencias concretas que pudieran surgir tanto por categorías académicas como por áreas de conocimiento.

Consideramos que dando respuesta a estos objetivos contribuiremos a clarificar una pequeña parte de nuestra herencia como investigadores: recordar en qué formatos han generado impacto nuestras investigaciones más citadas, comparar la realidad de las diferentes áreas que componen la etiqueta generalista de Comunicación y preguntarnos por las maneras en las que diferentes figuras académicas ya asentadas han configurado, en el paisaje del "Efecto ANECA", su producción más relevante.

\section{METODOLOGÍA}

\subsection{Muestra y procedimiento de recogida de datos.}

Durante la primera fase de la investigación (entre abril y mayo de 2019), se realizó un cribado de todos los departamentos de las universidades públicas españolas que cuentan con estudios de comunicación audiovisual, publicidad y/o periodismo. Para ello, se utilizaron las webs institucionales, si bien es necesario señalar que no todas las instituciones mantienen la información 
actualizada, accesible y bien estructurada -véase la crítica expuesta en Delgado Lopez-Cózar y otros, 2014- por lo que en diferentes ocasiones se tuvo que recurrir a contactos directos con las diferentes secretarías académicas. Se excluyeron aquellos centros que, pese a su denominación, no contaban todavía con catedráticos y titulares en estudios específicos de comunicación -siendo la investigación generada por el claustro en otros campos más o menos afines como educación, filologías o documentación-.

Una vez concluida esta primera fase, se contó con un universo total de cerca de 400 catedráticos y titulares repartidos entre las diferentes universidades públicas, sobre las que a su vez se aplicó un segundo filtro con los siguientes criterios excluyentes:

a. Los docentes e investigadores debían tener, al menos, tres trabajos diferentes que contaran con un mínimo de una cita.

b. Los docentes e investigadores debían contar con un perfil consultable en alguna de las siguientes webs: Google Scholar, Dialnet, Academia.edu o Researchgate que nos permitiera rastrear correctamente su actividad investigadora.

c. Los docentes e investigadores debían trabajar específicamente en el campo de la comunicación.

Una vez aplicado el segundo filtro, la muestra quedó compuesta por 281 autores (82 catedráticos, 199 titulares), cuyo reparto quedará desplegado en el apartado 3.1. A partir de dicha muestra se abordó la extracción de datos (junio a agosto de 2019) tomando como referencia los datos de citación ofrecidos por Google Scholar Citations. La elección de este indicador se basa en diversos estudios que han puesto de manifiesto que Google Scholar ofrece unos índices de impacto más altos, menor sesgo anglosajón y mayor cobertura de fuentes documentales que Web of Science y Scopus (Degado López-Cózar y Repiso, 2013; Orduña-Malea y Delgado López-Cózar, 2014; Martín-Martín y otros, 2018). Del mismo modo, el estudio concreto de libros y artículos utilizando los indicadores de Google Scholar cuenta con referentes notables en el campo (Gantman, Dabós, 2018; Martín-Martín, Orduna-Malea, Ayllón, Delgado López-Cózar, 2016) que serán tenidas en cuenta a la hora de redactar el análisis de los resultados de la presente investigación. Por "casos de éxito" entendimos aquellas tres publicaciones que arrojaban un mayor número de citas en cada perfil individual.

Como es bien sabido, la fiabilidad en el cómputo de las citas que recogen los perfiles de Google Scholar, no siempre arroja la necesaria exactitud. Pensamos, por ejemplo, en las llamadas "citas ex- traviadas" que pueden replicar una misma publicación con varios registros, por un problema ortográfico o por otro tipo de incidencia en la indexación -tema que ya ha contado con una exhaustiva revisión bibliográfica (Orduña-Malea y otros, 2017)-. Para compensar este problema, en absoluto menor, se han revisado manualmente los perfiles estudiados, cotejándolos con la información disponible en otras bases de datos como Researchgate, Academia.edu y Dialnet.

En el caso de aquellos profesionales que no contaban con el perfil abierto en Google Scholar Citations $(\mathrm{N}=86,36 \%)$ se utilizó una búsqueda manual de referencias tomando como base la información de su perfil en la página web de su universidad de origen, cotejada a su vez con los datos ofrecidos por Dialnet+ y, finalmente, con el cálculo de citas arrojado por la búsqueda manual en Google Scholar. Debido a la notable velocidad con la que se actualizan los resultados en dichas plataformas se realizó un segundo cotejo de las citas la semana antes de concluir la redacción del artículo (tercera semana de septiembre de 2019) para asegurar la mayor fiabilidad en los mismos. Todos los procesos estadísticos fueron realizados mediante el programa SPSS v. 25.

\subsection{Instrumento de recogida de información}

Siguiendo los tres objetivos marcados en la Introducción del presente artículo, decidimos diseñar una herramienta ad hoc. La herramienta queda referenciada en la Tabla I.

La primera área se vincula con la sistematización del campo, tal y cómo es habitual en este tipo de trabajos. La segunda área selecciona, a su vez, los tres trabajos más citados (II.1, II. 2 y II.3) y se divide, a su vez, la atención en el canal de publicación (Ítems II.1/2/3.a) y en el número de citas conseguido por dicho trabajo (Ítems II.1/2/3.b), por lo que podremos acceder con facilidad a los casos con mayor impacto por investigador. En este caso, quedará vinculada al objetivo de investigación 1 . Por último, la tercera área propondrá un análisis global de la muestra, mostrando las diferencias -si las hubiera- entre categorías profesionales y campos epistemológicos. En este caso, nos proponemos preguntarnos, aunque sea someramente, sobre la problemática que emerge en dos campos claves para nuestra actividad investigadora:

a. En primer lugar, las diferencias en la manera de concebir la investigación que ha disparado el ya citado "efecto ANECA" como las diferentes políticas de acceso a las acreditaciones de cuerpos docentes (Ítem III.01), y que por lo tanto ha generado diferentes aproximaciones a los for- 
Tabla I: Variables medibles. División en áreas de interés e ítems.

\begin{tabular}{|c|c|c|}
\hline ÁREA & ÍTEM MEDIDO & \\
\hline \multirow{3}{*}{ I. Identificación } & 01. Universidad de origen del investigador & \\
\hline & 02. Categoría profesional (Catedrático / Titular) & \\
\hline & $\begin{array}{l}\text { 03. Área principal de investigación (Comunicación } \\
\text { Audiovisual y Publicidad / Periodismo) }\end{array}$ & \\
\hline \multirow{6}{*}{$\begin{array}{l}\text { II. Estudio individual de } \\
\text { casos de éxito }\end{array}$} & \multirow{2}{*}{ 01. Trabajo más citado } & a. Tipo de publicación \\
\hline & & b. Número de citas \\
\hline & \multirow{2}{*}{ 02. Segundo trabajo más citado } & a. Formato de publicación \\
\hline & & b. Número de citas \\
\hline & \multirow{2}{*}{ 03. Tercer trabajo más citado } & a. Formato de publicación \\
\hline & & b. Número de citas \\
\hline \multirow{2}{*}{$\begin{array}{l}\text { III. Estudio global de } \\
\text { casos de éxito }\end{array}$} & $\begin{array}{l}\text { 01. Casos de éxito por categoría investigadora } \\
\text { (Catedrático/Titular). }\end{array}$ & \\
\hline & $\begin{array}{l}\text { 02. Casos de éxito por área (Comunicación } \\
\text { Audiovisual y Publicidad / Periodismo) }\end{array}$ & \\
\hline
\end{tabular}

matos de publicación por parte de catedráticos y titulares.

b. El propio estatuto poliédrico y complejo de las ciencias de la comunicación -volveremos a esta idea en la discusión del presente artículo- arrastra, a su vez, una riqueza de objetos de estudio y campos de trabajo que requieren, a su vez, de una gran pluralidad de enfoques en la recogida de datos o la publicación de resultados. El hecho de que se encuentren divergencias entre campos indiscutiblemente conectados -al menos, como veremos, burocráticamente- como la Comunicación Audiovisual, la Publicidad y las Relaciones Públicas y el Periodismo podría suponer una invitación para repensar el funcionamiento y la valoración de los diferentes formatos de publicación académicos. Responderá a esta problemática el ítem III.02. En ambos casos, la tercera área responderá al segundo y tercer objetivo de investigación. Nos gustaría señalar, además que el índice de solapamiento entre los documentos extraídos es extraordinariamente bajo $(0.2 \% \mathrm{~N}=2)$, por lo que no hemos tenido en cuenta dicho rasgo a la hora de realizar la lectura de los datos.

Somos conscientes de que nuestro diseño metodológico deja voluntariamente fuera un indicador que sería, sin duda, especialmente relevante para retomar en futuras investigaciones: la fecha de publicación de cada uno de los ítems estudiados. Como ya han demostrado otros trabajos del campo (Túñez-López y otros, 2014), la tendencia en los estudios de comunicación ha impulsado una mayor citación de artículos de revistas en detrimento de los libros académicos. Sin poder desplegar aquí la profundidad que exige este debate, bien merece la pena realizar un breve apunte metodológico: al sistematizar los libros académicos citados hemos descubierto una notable pluralidad de rasgos, estilos, usos y finalidades que van desde el clásico manual hasta el ensayo de corte inequívocamente personal. No se nos escapa que ambos canales tienen una marcada diferencia epistemológica que, afortunadamente, de vez en cuando permite observar también ver hibridaciones, contaminaciones y tránsitos entre ambas: por un lado, el artículo suele diseñarse como un informe conciso y actualizado de una investigación acotada, mientras que el libro puede optar por una mirada divulgativa o directamente experimental sobre la experiencia científica (García-Catalán, 2012). De un libro se espera, al menos teóricamente, que consolide un conocimiento de mayor recorrido y que, en consecuencia, su influjo sobre la comunidad académica genere efectos de mayor recorrido. Saber si realmente estos ítems "de éxito" que manejamos tienen algún tipo de sesgo temporal nos permitiría analizar esa repercusión a largo plazo de todos los canales de comunicación y extraer consecuencias sobre la potencialidad de su citación -objetivo que no podemos sino apuntar aquí y dejar esbozado en espera de futuras publicaciones-.

\section{RESULTADOS Y DISCUSIÓN}

\subsection{Perfil de los investigadores}

Como ya se ha señalado, tras aplicar los criterios de selección determinados se ha trabajado con un total de 281 docentes e investigadores repartidos según se observa en la tabla II. 
Tabla II: Muestra por universidades y categorías profesionales.

\begin{tabular}{|c|c|c|}
\hline Universidad de origen & Catedráticos & Titulares \\
\hline Universidad Autónoma de Barcelona & 7 & 3 \\
\hline Universidad de Alicante & 1 & 13 \\
\hline Universidad de Barcelona & 2 & 1 \\
\hline Universidad Carlos III & 3 & 20 \\
\hline Universidad de Cádiz & 0 & 6 \\
\hline Universidad Complutense de Madrid & 20 & 2 \\
\hline Universidad de Elche & 1 & 0 \\
\hline Universidad de Extremadura & 0 & 3 \\
\hline Universidad de Girona & 1 & 0 \\
\hline Universidad de Granada & 1 & 2 \\
\hline Universitat Jaume I & 5 & 1 \\
\hline Universidad de La Laguna & 0 & 2 \\
\hline Universidad de Málaga & 6 & 16 \\
\hline Universidad de Murcia & 1 & 4 \\
\hline Universidad del País Vasco & 3 & 13 \\
\hline Universitat Pompeu Fabra & 7 & 12 \\
\hline Universitat Politécnica de València & 1 & 1 \\
\hline Universidad Rey Juan Carlos & 7 & 45 \\
\hline Universidad Rovira i Virgili & 0 & 4 \\
\hline Universidad de Salamanca & 1 & 3 \\
\hline Universidad de Santiago de Compostela & 5 & 4 \\
\hline Universidad de Sevilla & 5 & 27 \\
\hline Universidad de València & 4 & 12 \\
\hline
\end{tabular}

Como era esperable, la propia disparidad en los tamaños de los diferentes campus y departamentos, así como las propias fortalezas y dificultades de cada comunidad autónoma en los procesos de consolidación de plazas destinadas al funcionariado de las universidades ha conformado una muestra extraordinariamente heterogénea. En este sentido, es llamativa la presencia de PDI consolidado con trabajos citados en los campus más jóvenes de la Comunidad de Madrid (Universidad Rey Juan Carlos - 18,5\%, Universidad Carlos III - 8,2\%) y de las universidades andaluzas (Universidad de Sevilla - $11,4 \%$ y Universidad de Málaga - 7,8\%).

Un dato que consideramos digno de mención es que prácticamente la totalidad de las trayectorias profesionales estudiadas contaban con libros y revistas entre sus casos de éxito. No obstante, encontramos 28 excepciones que llamaron nuestra atención: 23 investigadores no habían recibido ninguna cita por su participación en libros, mientras que otros 5 no contaban en su haber con ninguna publicación en una revista académica que arrojara una cita. A nuestro juicio, no deja de ser una anecdótica pero brillante prueba de la pluralidad y heterogeneidad con la que se han configurado en los últimos años los perfiles académicos en el campo de la comunicación: al comprobar los intereses profesionales de dichos investigadores, pudimos detectar que las anomalías en sus trayectorias eran totalmente coherentes con la línea general con la que habían trazado su investigación, ya fuera por lo específico de ciertos campos que parecen exigir la extensión de la monografía específica -historia del cine y de la televisión, antropología de la imagen, teoría de la imagen, narrativa audiovisual, etc.- o, por el contrario, por aquellos intereses cuya urgencia y radical novedad recomiendan la publicación de estudios con la menor demora posible -audiencias, tecnología de la comunicación, actualidad política, redes sociales, etc.

Pese a que recuperaremos esta idea al final del artículo, veamos por el momento cómo han quedado dispuestos los datos individuales de cada uno de los 281 participantes estudiados. 


\subsection{Trabajos con mayor citación}

A la hora de estudiar a cada uno de los investigadores se decidió tomar como referencia cada uno de los tres trabajos con mayor impacto para evaluar, en lo posible, cuáles eran los formatos de publicación que habían ofrecido mayor rendimiento a las categorías académicas españolas en el campo de la comunicación. Veamos, por lo tanto, cómo queda dispuesta la fotografía general (tabla III). porque los temas dejan de resultar relevantes para la comunidad académica, bien por la propia caducidad y evanescencia de los datos recogidos y las conclusiones ofrecidas -y ninguna prueba mejor que la que el lector tiene ahora mismo entre manos: las citas en Google Scholar se actualizan prácticamente en tiempo real-. En contraposición, el libro académico tiene una larga tradición, en primer lugar, formativa -mejor que peor, al investigador cualificado

Tabla III: Trabajos más citados por formato de publicación y conjunto total de citas arrojadas por los 843 ítems más exitosos de catedráticos y titulares españoles en el área de comunicación.

\begin{tabular}{|l|c|c|c|c|c|}
\hline Formato de publicación & $\begin{array}{c}\text { Trabajo más } \\
\text { citado 1 }\end{array}$ & $\begin{array}{c}\text { Trabajo más } \\
\text { citado 2 }\end{array}$ & $\begin{array}{c}\text { Trabajo más } \\
\text { citado 3 }\end{array}$ & Total & $\begin{array}{c}\text { Número de citas } \\
\text { arrojadas }\end{array}$ \\
\hline Libro & 170 & 137 & 114 & 420 & 53416 \\
\hline Revista & 107 & 138 & 158 & 402 & 18475 \\
\hline Informe profesional & 1 & 4 & 5 & 10 & 781 \\
\hline Tesis doctoral & 2 & 1 & 0 & 3 & 31 \\
\hline Acta de congresos & 1 & 1 & 4 & 5 & 290 \\
\hline TOTAL & $\mathbf{2 8 1}$ & $\mathbf{2 8 1}$ & $\mathbf{2 8 1}$ & $\mathbf{8 4 3}$ & $\mathbf{7 2 9 9 3}$ \\
\hline
\end{tabular}

Lo primero que llama la atención es que, si bien el volumen de libros $\left(F_{1}=420,50 \%\right)$ y revistas $\left(F_{2}=402\right.$, $48 \%$ ) citadas parece mantener un cierto equilibrio, su rendimiento cualitativo es bien diferente. El total de citas arrojadas por libros es casi tres cuartas partes $\left(F_{1}=53416,73 \%\right)$ del total estudiado, quedando las revistas académicas $\left(F_{2}=18475,25 \%\right)$ en una posición mucho más discreta (figura 1 ).

Figura 1: Comparación de ítems con mayor éxito y citas arrojadas.

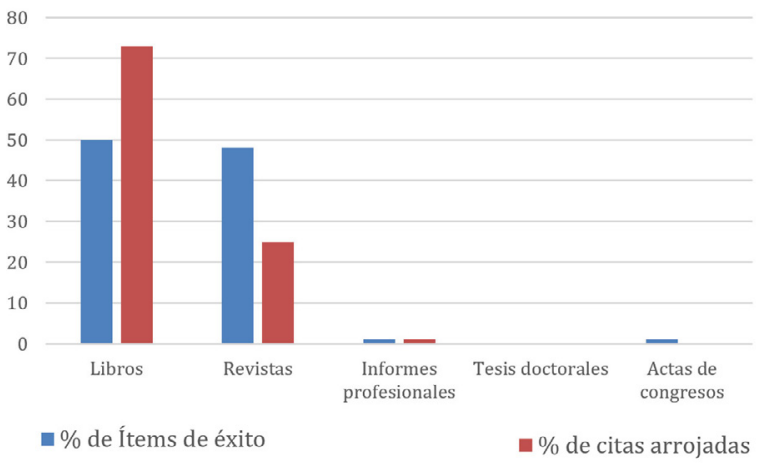

Sin duda, son muchas las razones que se podrían intuir para esta descompensación: si bien el artículo científico responde con precisión y claridad a un problema concreto, en demasiadas ocasiones tiene un recorrido intelectual mucho más corto: bien sea se le presupone el haberse empapado de los textos básicos de su disciplina-. En segundo lugar, al buen libro se le presupone también ser el fruto de una investigación personal y profesional de largo recorrido que exige un tiempo, una concentración y una madurez en su despliegue que no siempre se puede traducir con facilidad al formato paper.

Podemos, además, señalar otros datos de interés: el impacto absolutamente irrelevante de las tesis doctorales -con apenas 3 casos de éxito- y, muy especialmente, de las actas de congresos -5 casos de éxito-. En este último caso debemos señalar que en los cinco casos estudiados se trató de congresos anglosajones, firmados por más de seis autores, escritos en inglés y de temática muy específica (redes sociales, y convergencia mediática, principalmente).

Añadamos, además, algunos datos que pueden clarificar todavía más la fotografía propuesta del campo. En primer lugar, dentro de la categoría $\mathrm{Li}$ bros $(\mathrm{N}=420)$ podemos destacar que las monografías específicas $(\mathrm{N}=285)$ superaban a los capítulos de libro $(\mathrm{N}=135)$. Del mismo modo, y tomando en consideración todos los formatos de publicación, se puede señalar que la lengua de producción en casos de éxito seguía siendo mayoritariamente el castellano $(\mathrm{N}=726)$, seguida del inglés $(\mathrm{N}=98)$, lengua que se apreciaba especialmente en papers en revistas anglosajonas y capítulos de libro. Las otras dos lenguas en las que se han detectado ca- 
sos de éxito han sido el portugués $(\mathrm{N}=14)$ y el catalán $(\mathrm{N}=5)$.

\subsection{Citación por disciplinas: Comunicación Audiovisual y Publicidad vs. Periodismo}

A la hora de analizar los perfiles de los investigadores, se ha tomado como referencia su pertenencia a departamentos vinculados, bien con el área de Comunicación Audiovisual y Publicidad, bien con el área de Periodismo. Aunque es común encontrar perfiles híbridos dentro de cada departamento, de las 25 universidades estudiadas hemos localizado trece en la que conviven de manera más o menos equilibrada ambas ramas del campo, nueve centradas específi- camente en Comunicación Audiovisual y Publicidad y 4 más enfocadas en los estudios de periodismo. El desglose de los 281 perfiles estudiados arrojaba que 155 (43 catedráticos y 112 titulares) formaban parte de la primera categoría y 126 (39 catedráticos y 87 titulares) de la segunda. Dicho esto, la distribución en campos, formatos y citas queda configurada como puede verse en la Tabla IV.

Pese a la ligera diferencia del volumen de ambas áreas, parece claro que no se detectan diferencias reseñables en ambos campos. Una formulación visual (Fig. 2) permite comprobar cómo en ambos casos se mantienen volúmenes de citación similares y el mismo equilibrio en lo que toca a los formatos de publicación.

Tabla IV: Casos de éxito y número de citas arrojadas en las áreas de Comunicación Audiovisual y Publicidad y Periodismo.

\begin{tabular}{|c|c|c|}
\hline \multicolumn{3}{|c|}{ Investigaciones del área de Comunicación Audiovisual y Publicidad } \\
\hline Formato de publicación & No de items de éxito & No de Citas recogidas \\
\hline Libro & 231 & 28052 \\
\hline Revista & 224 & 8502 \\
\hline Informes & 4 & 274 \\
\hline Congreso & 3 & 47 \\
\hline Tesis doctoral & 3 & 31 \\
\hline Total & 465 & 36906 \\
\hline \multicolumn{3}{|c|}{ Investigaciones del área de Periodismo } \\
\hline Formato de publicación & No de items de éxito & No de Citas recogidas \\
\hline Libro & 190 & 25364 \\
\hline Revista & 179 & 9973 \\
\hline Informes & 6 & 507 \\
\hline Congreso & 3 & 243 \\
\hline Tesis doctoral & 0 & 0 \\
\hline Total & 378 & 36087 \\
\hline
\end{tabular}

Figura 2: Comparación de Ítems de éxito y citas arrojadas por área.

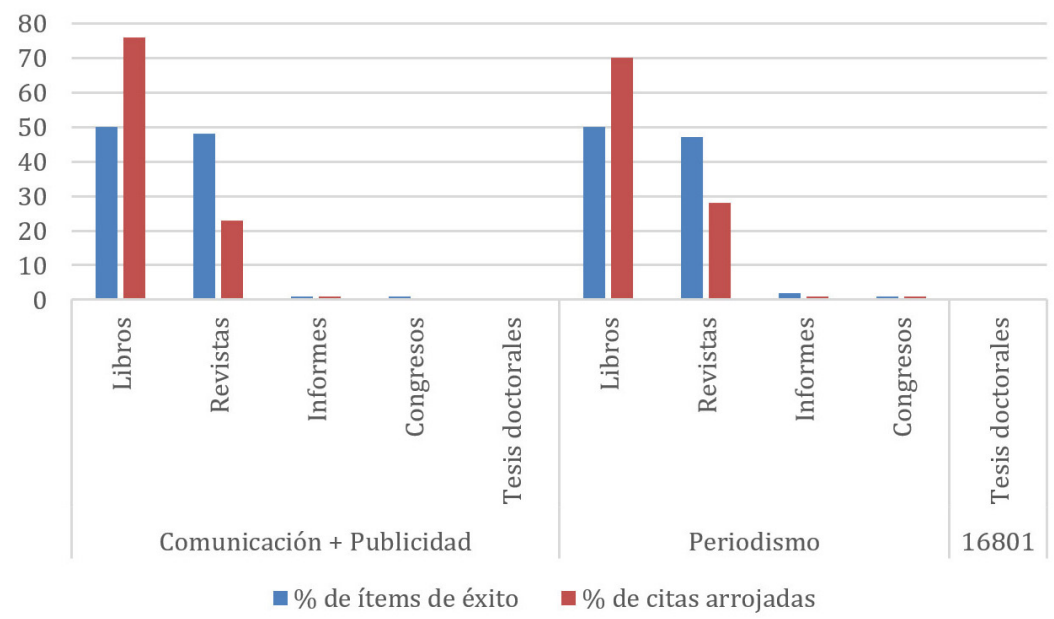


De manera puramente simbólica, puede señalarse una ecuanimidad indudable en el campo del libro entre los estudios audiovisuales $\left(F_{1}=231,50 \%\right)$ y periodismo $\left(F_{7}=190,50 \%\right)$ que muestra una brevísima desviación en lo que al número total de citas arrojadas se refiere $\left(F_{1}=28052,76 \%\right.$ frente a $\left.\mathrm{F}_{7}=25364,70 \%\right)$. El caso concreto de las revistas es algo diferente, en tanto si bien se detecta también una relevancia similar en los casos de éxito $\left(F_{2}=224,48 \%\right.$ frente a $\left.F_{8}=179,47 \%\right)$, sí que se experimenta una modificación algo más sustanciosa en el rendimiento en citas que arroja cada uno de los ítems $\left(F_{2}=8502,23 \%\right.$ frente a $\left.F_{8}=9973,28 \%\right)$.

\subsection{Citación por categorías profesionales: Ca- tedráticos frente a Titulares.}

Como ya se apuntaba al comienzo del artículo, uno de nuestros objetivos es detectar las posibles divergencias en cuanto a la relación entre formatos de publicación, número de citación y categoría profesional. Como ya se ha señalado en diferentes lugares (Giménez, 2016), la investigación en nuestro campo ha sufrido no pocas modificaciones en las últimas décadas que, en el constante baile de baremos, puntuaciones, exigencias de las agencias de acreditación y otros factores sobradamente conocidos, han modificado los intereses, esfuerzos y voluntades de los jóvenes investigadores.

Más allá de las preferencias individuales o de las características concretas de cada subdisciplina, parece evidente que en el momento de redactar estas líneas la preminencia que ocupan los papers en el debate sobre la gestión de la investigación está fuera de toda duda, dejando más bien la generación de libros académicos en un plano puramente vocacional y anecdótico. Veamos, en primer lugar, cuál es la relación simplificada entre dicha corriente y las cifras concretas arrojadas por las investigaciones de éxito (tabla V).
En efecto, el primer dato que llama la atención es la diferencia que se establece en el propio volumen de los formatos de publicación. En el caso de los catedráticos, una notable mayoría de las aportaciones exitosas $\left(F_{1}=164,67 \%\right)$ reside en el trabajo publicado en el libro. Más crudo es todavía el indicador de la cantidad de citación: menos de un quinto de las citas recogidas a lo largo de su trayectoria $\left(F_{2}=7931,19 \%\right)$ son fruto del trabajo publicado en revistas académicas.

Al descender a la columna de los titulares, la tendencia se invierte, al menos en cuanto a la cantidad de los trabajos relevantes. Las revistas son aquí las protagonistas -con un $23 \%$ de índice de éxito al comparar los valores en $F_{2}$ y $F_{7}$ ) - si bien también podemos comprobar que de dicho aumento no se sigue exactamente su correspondiente correlación en el número de citas recibidas por trabajo. Las revistas aumentan un $15 \%$ la cantidad de citas recibidas -véase, de nuevo, la diferencia entre $F_{2}$ y $\mathrm{F}_{7}-$, manteniendo los libros el $64 \%$ de la cantidad de citas que han engrosado sus respectivos índice $h$.

Más allá de poder confirmar la creciente relevancia de las revistas académicas entre los profesores titulares que optan a futuras cátedras de investigación, consideramos pertinente también realizar un breve repaso diferenciado por áreas de conocimiento dentro de las ciencias de la comunicación (tabla VI):

La tabla VI muestra, a grandes rasgos, que las cifras recogidas en el cómputo global se mantienen también en la división por áreas. Las revistas dominan por igual en el número de caso de éxito de los titulares -si bien, con una cierta superioridad tanto en casos de éxito como en citas recogidas en el área de Comunicación Audiovisual y Publicidad-, pero los volúmenes y los rasgos generales tienden, por lo demás, a mantenerse.

Tabla V: Comparativa de formatos de citación y citas en casos de éxito por las categorías de catedrático y titular.

\begin{tabular}{|c|l|c|c|c|c|}
\hline $\begin{array}{c}\text { Categoría } \\
\text { profesional }\end{array}$ & $\begin{array}{c}\text { Formato de } \\
\text { publicación }\end{array}$ & Casos de éxito & $\begin{array}{c}\text { Porcentaje de } \\
\text { casos de éxito }\end{array}$ & Citas recogidas & $\begin{array}{c}\text { Porcentaje de } \\
\text { citas recogidas }\end{array}$ \\
\hline \multirow{4}{*}{ Catedrático } & Libro & 164 & $67 \%$ & 33446 & $80 \%$ \\
\cline { 2 - 6 } & Revista & 78 & $31 \%$ & 7931 & $19 \%$ \\
\cline { 2 - 6 } & Informes & 4 & $2 \%$ & 331 & $1 \%$ \\
\cline { 2 - 6 } & Congreso & 0 & - & 0 & - \\
\cline { 2 - 6 } & Tesis doctoral & 0 & - & 0 & - \\
\hline \multirow{4}{*}{ Titular } & Libro & 257 & $43 \%$ & $1997019 \%$ & $34 \%$ \\
\cline { 2 - 6 } & Revista & 325 & $54 \%$ & 10544 & $1 \%$ \\
\cline { 2 - 6 } & Informes & 6 & $1 \%$ & 450 & $1 \%$ \\
\cline { 2 - 6 } & Congreso & 6 & $1 \%$ & 290 & - \\
\cline { 2 - 6 } & Tesis doctoral & 3 & $1 \%$ & 31 & \\
\hline
\end{tabular}


Tabla VI: Comparativa de formatos de citación y citas en casos de éxito según área por las categorías de catedrático y titular.

\begin{tabular}{|c|c|c|c|c|}
\hline $\begin{array}{c}\text { Categoría } \\
\text { profesional }\end{array}$ & Área & $\begin{array}{l}\text { Formato de } \\
\text { publicación }\end{array}$ & Casos de éxito & Citas recogidas \\
\hline \multirow{10}{*}{ Catedrático } & \multirow{5}{*}{$\begin{array}{l}\text { Comunicación Audiovisual } \\
\text { y/o Publicidad }\end{array}$} & Libro & 93 & 16801 \\
\hline & & Revista & 34 & 2442 \\
\hline & & Informes & 2 & 208 \\
\hline & & Congreso & 0 & 0 \\
\hline & & Tesis doctoral & 0 & 0 \\
\hline & \multirow{5}{*}{ Periodismo } & Libro & 71 & 16645 \\
\hline & & Revista & 44 & 5489 \\
\hline & & Informes & 2 & 123 \\
\hline & & Congreso & 0 & 0 \\
\hline & & Tesis doctoral & 0 & 0 \\
\hline \multirow{10}{*}{ Titular } & \multirow{5}{*}{$\begin{array}{l}\text { Comunicación Audiovisual } \\
\text { y/o Publicidad }\end{array}$} & Libro & 138 & 11251 \\
\hline & & Revista & 190 & 6060 \\
\hline & & Informes & 2 & 66 \\
\hline & & Congreso & 3 & 47 \\
\hline & & Tesis doctoral & 3 & 31 \\
\hline & \multirow{5}{*}{ Periodismo } & Libro & 119 & 8719 \\
\hline & & Revista & 135 & 4484 \\
\hline & & Informes & 4 & 384 \\
\hline & & Congreso & 3 & 243 \\
\hline & & Tesis doctoral & 0 & 0 \\
\hline
\end{tabular}

\section{DISCUSIÓN}

Como señalábamos en la introducción del artículo, el campo de la comunicación se encuentra inmerso en un momento de replanteamiento sobre su propio relato epistemológico. En este proceso de duda sobre el marco contextual de la evolución de la producción científica en comunicación, es necesario destacar la influencia de la Agencia Nacional de Evaluación de la Calidad y Acreditación (ANECA) que ha generado el llamado "Efecto ANECA" (Soriano, 2008). Muchas facetas del "Efecto ANECA" ya han sido sobradamente estudiadas por nuestros colegas. Así, se han explorado sus efectos sobre la autoría (Soriano, 2008, 2017; Fernández-Quijada y Masip, 2013), sobre la manera en la que ha desvelado los sesgos de género de nuestro campo (Martín-Algara y otros, 2018), o sobre sus efectos nocivos sobre la autonomía universitaria (González Requena, 2018). Pero también, en una dimensión más productiva, encontramos trabajos que versan sobre las complejas investigaciones coordinadas que ha propiciado (Coffarel-Serra y otros, 2018; Coffarel-Serra y otros, 2017; Diaz-Nosti y De Frutos, 2017; Tur-Viñes y Núñez-Gómez, 2018), sobre el aumento exponencial y la internacionaliza- ción de la producción científica en nuestro campo (Masip, 2011) o sobre los nuevos retos en los procesos evaluativos (Codina, 2016). Este contexto -cuyas consecuencias son quizá menos perceptibles en otras facetas de nuestra actividad profesional como por ejemplo en la evaluación de los sexenios de investigación- ha generado no pocos problemas, siendo quizá los más urgentes la precarización del tejido académico (Castillo-Alonso y Moré, 2016) y la aplicación de medidas neoliberales a la gestión del conocimiento (Marzal-Felici y otros, 2018). También se han dedicado estudios parciales a diferentes campos como la publicidad (Baladrón-Pazos y otros, 2017; Correyero-Ruiz y otros, 2018), el análisis fílmico (Rodríguez-Serrano y otros, 2019) o la radio (Piñeiro-Otero, 2016, 2018), demostrando que el campo de estudios sobre comunicación sigue siendo heterogéneo y de tan inmensa complejidad que las tesis esbozadas al respecto hace más de una década por Leonarda García-Jiménez (2007) siguen plenamente vigentes. Aunque parezca una simple cuestión anecdótica, durante la extracción de datos nos percatamos de la tremenda confusión que existe para catalogar nuestra propia actividad como investigadores, per- 
judicando así nuestra capacidad para atraer citas y para difundir los resultados de nuestras investigaciones. Como es bien sabido, Google Scholar permite incorporar al menos una palabra clave que sirva para localizar el campo de acción de cada perfil. Nosotros recogimos un total de 627 palabras clave -alrededor de una media de 3,2 etiquetas usadas por investigador-. Un dato especialmente interesante es que el $7,7 \%$ de los perfiles analizados prescindieron de cualquier tipo de Keyword en su presentación a la comunidad académica, resultando así confusa su posición dentro del tablero epistemológico. Esa tremenda heterogeneidad del campo generaba, a su vez, una indudable dispersión de los contenidos: De las 627 palabras procesadas, 237 (un 38\%) aparecen una única vez y cubren aspectos que muestran investigaciones muy especializadas. Al contrario, las tres categorías que se repiten un mayor número de veces son la genérica Comunicación $(\mathrm{N}=55)$, y dos de las ramas mayores, Periodismo $(\mathrm{N}=37)$ y Publicidad $(\mathrm{N}=21)$.

Dejando a un lado este rasgo urgentemente mejorable de nuestra disciplina, creemos que nuestros resultados son, a grandes rasgos, coherentes con los de los autores que hemos citado a lo largo del presente trabajo: nuestro estudio arroja una convivencia entre formatos, si bien desvela también las consecuencias perversas del llamado "Efecto ANE$\mathrm{CA}^{\prime \prime}$ (necesidad de impacto más rápido a coste del alcance temporal de cada propuesta, especialmente entre profesores titulares), así como la inflación del número de artículos publicados en relación con las citas recibidas.

Ahora bien, si comparamos nuestros resultados con los del marco internacional según lo señalado por algunos de los trabajos consultados (Martín-Martín y otros, 2016), vemos que la tendencia española en el campo concreto de la comunicación es diferente a la fotografía general, especialmente en el campo de las ciencias de la naturaleza o experimentales: allí la primacía del artículo es incuestionable. Ahora bien, al hilo de este estudio, es necesario señalar que en la colección de los veinticinco trabajos más citados desde 1950 no hay ni un ejemplo propio de nuestro campo, siendo lo más cercano trabajos propios de la psicología, de la biblioteconomía, de la metainvestigación o de las ciencias políticas. En todos estos casos, además, se trata de libros y no de artículos científicos. Por otro lado, si atendemos al volumen de publicaciones de éxito, vemos que los marcos en comunicación son muy similares a los de otras disciplinas afines. Por ejemplo, estudios comparativos como el ya citado de Gantman y Dabós (2018) señalan que en sociología el volumen de artículos relevantes es del $47,85 \%$-en comuni- cación hemos detectado un 50\%- mientras que en otras disciplinas como economía o marketing se sitúa en un $46 \%$ y un $55 \%$, respectivamente. Volviendo a nuestro caso, se observa una mayor diferencia a la hora de realizar los cálculos de rentabilidad de cada ítem exitoso: los libros arrojan a la larga un número casi tres veces mayor de citas que los papers.

\section{CONCLUSIONES}

Aunque resulte paradójico, en pleno 2019 y en el campo concreto de los estudios de comunicación, la opacidad sigue siendo un rasgo notable en la constitución de los departamentos universitarios. Además de las dificultades encontradas para acceder a los organigramas y cargos de los miembros de cada departamento, hay que añadir un notable descuido en la centralización y consulta de los datos correspondientes a la investigación -webs institucionales sin actualizar, perfiles académicos eliminados y, en el límite, profesores con alta cualificación académica que no contaban con trabajos citados o con más de tres publicaciones indexadas, ni siquiera en Dialnet-. Este rasgo da buena cuenta de la ausencia de criterios verificables, transparentes y objetivos que ha operado hasta hace bien poco en la selección de funcionarios a los cuerpos docentes. Además, una parte importante de los perfiles estudiados (36\%) no contaban con perfil abierto en Google Scholar. Pese a todo, creemos que la muestra manejada (281 investigadores, 843 trabajos estudiados) ha sido suficientemente valiosa y heterogénea. Una vez alcanzado este punto, podemos retomar los objetivos principales de nuestra investigación para sintetizar los siguientes resultados:

R1: El análisis individual de los tres casos de mayor éxito por cada investigador demostró que, en una primera aproximación, libros y revistas académicas conviven arrojando un porcentaje similar. Se constata, además, la práctica irrelevancia en el panorama nacional de las actas de congresos, las tesis doctorales o los informes profesionales. Dentro de la categoría Libros, se observa una mayor citación en las monografías que en los capítulos. La lengua mayoritaria sigue siendo el castellano, si bien también se localizan casos de éxito en inglés, portugués y catalán.

R2: No se observan diferencias relevantes entre los resultados propios de las áreas Comunicación Audiovisual y Publicidad frente a Periodismo. Se puede concluir que en ambos casos se sigue un patrón muy similar al cómputo global del campo.

R3: Se observa una cierta tendencia de cambio cuando se comparan los resultados de citación de 
catedráticos y titulares de universidad. En el caso de las figuras con mayor rango académico, se constata un mayor interés por el libro, pero, sobre todo, un número mayor de citas cosechadas en dicho formato de publicación durante su carrera. En el caso de los profesores titulares, el volumen de publicaciones situadas en revistas académicas aumenta muy notablemente; sin embargo, el índice de citas arrojadas por los libros sigue siendo sensiblemente mayor. En este cambio de tendencia ha debido influir el hecho de que la mayoría de comisiones del ámbito de las ciencias sociales y jurídicas de la Comisión Nacional de Evaluación de la Actividad Investigadora (CNEAI) o del Programa Academia de ANECA otorgan muy poco valor al libro como formato de publicación científico, un claro "efecto ANECA".

Somos conscientes de las limitaciones que nuestra investigación presenta. El propio concepto de citación, por la propia inflexibilidad de la mera suma cuantitativa, deja fuera de su rango factores tan extraordinariamente importantes $-y$, generalmente, tan poco valorados- como la implicación personal ante la investigación y la docencia, el valor docente - la pura transferencia de los trabajos publicados (Recalcati, 2016), o la relevancia social concreta que una investigación arroja sobre un contexto de desigualdad o de injusticia, local o universal. Un mayor número de citas no garantiza, obviamente, la manera en la que dicho trabajo repercute en campos que realmente requieren un impacto (humano) como podrían ser la accesibilidad tecnológica, la inmigración, la gestión de la memoria histórica o la representación de los colectivos en riesgo de exclusión. Un buen ejemplo de esto nos sale al paso al pensar en el poco "impacto directo" de las tesis doctorales que arrojan nuestros indicadores, mientras que en la mayoría de los casos sus efectos "indirectos" - desde los que afectan al aprendizaje, a la ética de la investigación o a la propia definición del perfil profesional- suelen ser mucho mayores.

Del mismo modo, la presente investigación viene a confirmar que cuando se trata de analizar la calidad de la producción científica en ámbitos como la investigación en comunicación -o en el marco general de las ciencias sociales y de las humanidades-, parece aconsejable seguir las recomendaciones enunciadas en la "Declaración de San Francisco de Evaluación de la Investigación" (DORA, 2012) o en el "Manifiesto de Leiden sobre indicadores de investigación" (Hicks y otros, 2015): cabe reconocer "las diferencias en las prácticas de publicación y citación entre campos científicos", al tiempo que algunos indicadores como el factor de citación o el índice $\mathrm{h}$ deben ser periódicamente analizados y actualizados.
La fotografía que arroja nuestra investigación muestra un panorama en el que revistas académicas y libros han convivido hasta el momento, perfectamente integradas en un ecosistema epistemológico en el que cada tema y cada investigación ha tenido la oportunidad de ocupar su espacio y su tiempo en el formato de investigación pertinente. No es, dicho sea de paso, un rasgo anecdótico de nuestro tejido: en la actualidad 5 de las 20 revistas más relevantes en JCR -Journal of Communication, The International Journal of Press/politics, Public Understanding of Science, Journalism y Mobile Media \& Communication- publican puntualmente $y$, de manera periódica, reseñas y aproximaciones críticas a los últimos libros publicados en nuestro campo. No obstante, la aceleración en la exigencia de los procesos de saber, nuestro "Efecto ANECA" o la preeminencia acrítica de las métricas por encima de la reflexión de largo recorrido parece apuntar, como han señalado otros trabajos (Túñez-López y otros, 2014), a que en el futuro este equilibrio puede correr peligro si no se respeta la pluralidad, la diversidad y el sentido común de nuestros investigadores, así como los recursos y naturalezas propias de cada departamento, de cada ámbito gnoseológico y de cada tema estudiado. Cualquier académico no cegado por la aparente claridad de sus hallazgos puede dar cuenta de que el factor de éxito de una publicación -al menos en unas ciencias que se reivindican sociales, y también, humanas- no pasa únicamente por el índice $\mathrm{h}$ que generan sino por su capacidad para formar, perdurar, matizar o, en definitiva, modificar el mundo -y exija el lector o lectora la profundidad que considere necesaria a dicha afirmación-.

En suma, la presente investigación ha querido visibilizar el papel clave jugado por las revistas académicas en los últimos años, pero también poner de relieve la relevancia del libro académico como formato de publicación, capaz de hacer avanzar la elaboración y difusión del conocimiento científico en nuestro campo. Creemos, además, que ya hay mecanismos de control y evaluación de contenidos que pueden garantizar el mismo rigor metodológico y el cumplimiento de unos estándares de calidad públicamente contrastables: el sello de calidad CEA-APG (Calidad en Edición Académica), impulsado por la Unión de Editoriales Universitarias Españolas, o el sistema de información SPI (Scholarly Publishers Indicators), desarrollado por el Grupo ILIA del CSIC, bajo la dirección de Giménez-Toledo y otros $(2012,2014,2018)$. Actuar con parámetros inclusivos y atentos a la diversidad en la investigación generará, a nuestro juicio, la necesaria pluralidad que requiere un campo tan heterogéneo, complejo y rico como el de la investigación en comunicación. 


\section{AGRADECIMIENTOS}

El presente estudio ha sido realizado con la ayuda del Proyecto de Investigación "Mapas de la Investigación en Comunicación en las universidades españolas de 2007 a 2018" (código PGC2018-093358-B-100), bajo la dirección de Carmen Caffarel Serra y Carlos Lozano Ascencio, financiado por la Convocatoria 2018 de Proyectos de I+D "Generación de Conocimiento", del Programa Estatal de Generación de Conocimiento y Fortalecimiento Científico y Tecnológico del Sistema I+D, en el Marco del Plan Estatal de Investigación Científica y Técnica y de Innovación del Ministerio de Ciencia, Innovación y Universidades, para el periodo 2019-2021. Asimismo, ha contado con el apoyo financiero del proyecto de investigación "Análisis de identidades discursivas en la era de la posverdad. Generación de contenidos audiovisuales para una Educomunicación crítica (AIDEP)", (código 18I390.01/1), bajo la dirección de Javier Marzal Felici, financiado por la Universitat Jaume I, y evaluado por la Agencia AGAUR, a través de la convocatoria competitiva de proyectos de investigación de la UJI, para el periodo 2019-2021.

Los autores quieren hacer público su reconocimiento al profesor Emilio Delgado López-Cózar, Catedrático de Biblioteconomía y Documentación de la Universidad de Granada, y de Lluís Codina, Profesor Titular de Biblioteconomía y Documentación de la Universitat Pompeu Fabra, por la ayuda prestada en el diseño y desarrollo de esta investigación. También deseamos manifestar nuestra

\section{REFERENCIAS}

Abadal, E. (ed.) (2017). Revistas científicas. Situación actual y retos de futuro. Barcelona: Universitat de Barcelona Edicions.

Baladrón-Pazos, A. J.; Manchado-Pérez, B.; Correyero-Ruiz, B. (2017). Estudio bibliométrico sobre la investigación en publicidad en España: temáticas, investigadores, redes y centros de producción (1980-2015). Revista Española de Documentación Científica, 40(2), pp. 1-18. https://doi.org/10.3989/redc.2017.2.1411

Caffarel-Serra, C.; Gaitán-Moya, J.; Lozano-Ascencio, C.; Piñuel-Raigada, J. L. (2018). Tendencias metodológicas en la investigación académica sobre Comunicación. Salamanca: Comunicación Social.

Caffarel-Serra, C.; Ortega-Mohedano, F.; Gaitán-Moya, J. (2017). Investigación en Comunicación en la universidad española en el período 2007-2014, El Profesional de La Información, 26(2), pp. 218-227. https://doi. org/10.3145/epi.2017.mar.08

Carrasco-Campos, Á.; Saperas Lapiedra, E. (2016). Cambio tecnológico, globalización neoliberal y hegemonías metodológicas en la investigación comunicativa inter- deuda a los evaluadores del presente artículo, cuya identidad desconocemos.

\section{AKNOWLEDGEMENTS}

This study has been carried out with the help of the Research Project "Mapas de la Investigación en Comunicación en las universidades españolas de 2007 a 2018" (PGC2018-093358-B-100), under the direction of Carmen Caffarel Serra and Carlos Lozano Ascencio, financed by the 2018 Call for R\&D Projects "Generation of Knowledge", of the State Program for Generation of Knowledge and Scientific and Technological Strengthening of the R\&D System, within the framework of the State Plan for Scientific and Technical Research and Innovation of the Ministry of Science, Innovation and Universities, for the period 2019-2021. It has also received financial support from the research project "Analysis of Discursive Identities in the Post-truth Era. Generation of Audiovisual Content for a Critical Educommunication (AIDEP)", (code 18I390.01/1), under the direction of Javier Marzal Felici, financed by the Universitat Jaume I, and evaluated by the AGAUR Agency, through the competitive call of UJI research projects, for the period 2019-2021.

The authors wish to acknowledge Professor Emilio Delgado López-Cózar, Professor of Library Science and Documentation at the University of Granada, and Lluís Codina, Professor of Library Science and Documentation at Pompeu Fabra University, for the help provided in the design and development of this research. We also wish to express our debt to the reviewers of this article, whose identity we do not know.

nacional. Ámbitos. Revista Internacional de Comunicación, 32, edición de primavera, pp. 1-9.

Carrasco-Campos, Á.; Saperas-Lapiedra, E.; Martínez-Nicolás, M. (2018). ¿Cómo investigamos la comunicación en España? Universidades públicas y privadas en las publicaciones científicas de comunicación españolas (1990-2014). AdComunica. Revista Científica de Estrategias, Tendencias e Innovación en Comunicación, (15), pp. 45-63. https://doi.org/10.6035/21740992.2018 .15 .4

Castillo-Alonso, J.; Moré, P. (2016). Por una sociología del trabajo académico: La precarización del trabajo de enseñar e investigar en la Universidad. Sociología Del Trabajo, (88), pp. 7-26.

Català, J. (2018). Sombras al final del túnel. La crisis del pensamiento en las ciencias de la comunicación. En: Rodríguez Serrano, A.; Gil Soldevilla, S. (eds.), Investigar en la era neoliberal. Visiones críticas sobre la investigación en comunicación en España. Barcelona, Bellaterra, Castellón y Valencia: Universitat Pompeu Fabra, Universitat Autònoma de Barcelona, Universitat Jaume I y Universitat de València, Col.lecció Aldea Global, pp. 33-54. 
Codina, L. (2016). Evaluación de la ciencia: Tan necesaria como problemática. El Profesional de la Información $(25,5)$, pp. 715-719.

Correyero-Ruiz, B.; Manchado-Pérez, B.; Baladrón-Pazos, A. (2018). Evolución histórica de la investigación publicitaria en España: de los orígenes científicos de la publicidad al actual auge investigador. AdComunica. Revista Científica de Estrategias, Tendencias e Innovación En Comunicación, (15), pp. 89-113. https://doi. org/10.6035/2174-0992.2018.15.6

Delgado López-Cózar, E.; Repiso, R. (2013). El impacto de las revistas de comunicación: comparando Google Scholar Metrics, Web of Science y Scopus. Comunicar. Revista Científica de Educomunicación, 41, Vol. XXI, 45-52. http://dx.doi.org/10.3916/C41-2013-04

Delgado López-Cózar, E.; Orduña-Malea, E.; Jiménez-Contreras, E.; Ruiz-Pérez, R. (2014). H Index Scholar: el índice $\mathrm{h}$ de los profesores de las universidades públicas españolas en humanidades y ciencias sociales. El profesional de la información, 23(1). https://doi.org/10.3145/epi.2014.ene.11

Díaz-Nosty, B.; De Frutos, R. (2017). Tendencias de la investigación universitaria española en Comunicación. Navarra: Thomson Reuters Aranzadi.

DORA (2012). "San Francisco Declaration on Research Assessment". https://sfdora.org

Fernández Quijada, D.; Masip, P. (2013). Tres décadas de investigación española en comunicación: hacia la mayoría de edad. Comunicar: Revista Científica Iberoamericana de Comunicación y Educación, (41), pp. 15-24. https://doi.org/10.3916/C41-2013-01

Gantman, Ernesto R.; Dabós, Marcelo P. (2018). Research output and impact of the fields of management, economics, and sociology in spain and france: An analysis using google scholar and scopus. Journal of the Association for Information Science and Technology, 69(8), pp. 1054-1066. http://doi.org/10.1002/asi.24020

García-Catalán, S. (2012). Solidarizar cuando en transformar no se cree: efectos de sentido y sinsentido en los discursos solidarios bajo el paradigma informativo. En: Proceedings of the 10th World Congress of the International Association for Semiotic Studies, pp. 1945-1954.

García-Jiménez, L. (2007). Las Teorías de la comunicación en España: un mapa sobre el territorio de nuestra investigación (1980-2006). Madrid: Tecnos.

Giménez-Toledo, E. (2016). Malestar. Los investigadores ante su evaluación. Madrid: Iberoamericana.

Giménez-Toledo, E.; Tejada-Artigas, C. (2012, 2014, 2018). Scholarly Publishers Indicators (SPI). $1^{\text {a }}$ Edición 2012; 2a Edición 2014; 3a Edición 2018. http:// ilia.cchs.csic.es/SPI/index.html

González Requena, J. (2018). La crisis de la universidad. En defensa de una verdadera autonomía universitaria. En: Bueno Doral, T. (ed.), Misión de la universidad en nuestros días. Un enfoque interdisciplinar. Libro - Homenaje al profesor Luis Gutiérrez-Espada. Valencia: Campgràfic, pp. 111-122.

Gorraiz, J. (2018). Los mil y un reflejos de las publicaciones en el laberinto de espejos de las nuevas métricas. El Profesional de La Información, 27(2), 231-236. https://doi.org/10.3145/epi.2018.mar.01
Hicks, Diana; W., Paul; W., Ludo; R., Sarah de; Rafols, I. (2015). The Leiden Manifest for Research Metrics, Nature, 520, pp. 429-431. http://www. leidenmanifesto.org

Martín-Algara, M.; Serrano-Puche, J.; Rebolledo, M. (2018). La mujer en la investigación en comunicación en España: un análisis de la producción científica (2007-2013). AdComunica. Revista Científica de Estrategias, Tendencias e Innovación En Comunicación, (15), pp. 65-87. https://doi.org/10.6035/21740992.2018.15.5

Martínez-Nicolás, M.; Carrasco-Campos, Á. (2018). La transformación de una comunidad científica. Evolución del patrón de autoría de la investigación española sobre comunicación publicada en revistas especializadas (1990-2014). Revista Latina de Comunicación Social, 73, 1368-1383. https://doi.org/10.4185/RLCS-20181311

Martínez-Nicolás, M.; Saperas, E.; Carrasco-Campos, Á. (2019). La investigación sobre comunicación en España en los últimos 25 años (1990-2014). Objetos de estudio y métodos aplicados en los trabajos publicados en revistas españolas especializadas. Empiria. Revista de Metodología de Ciencias Sociales, 42, pp. 37-69. https://doi.org/10.5944/empiria.42.2019.23250

Marzal-Felici, J.; Rodríguez-Serrano, A.; Gil-Soldevilla, S. (2018). Introducción. Reflexiones en torno a la naturaleza y a la calidad de la investigación en comunicación. Investigar en el contexto de la expansión del pensamiento neoliberal. En: Rodríguez Serrano, Aaron; Gil Soldevilla, Samuel (eds.), Investigar en la era neoliberal. Visiones críticas sobre la investigación en comunicación en España. Barcelona, Bellaterra, Castellón y Valencia: Universitat Pompeu Fabra, Universitat Autònoma de Barcelona, Universitat Jaume I y Universitat de València, Col.lecció Aldea Global, pp. 13-26.

Martín Algarra, M.; Serrano-Puche, J.; Rebolledo, M. (2018). La mujer en la investigación en comunicación en España: un análisis de la producción científica (20072013). adComunica. Revista Científica de Estrategias, Tendencias E Innovación En Comunicación, (15), 6587. http://doi.org/10.6035/2174-0992.2018.15.5

Martín-Martín, A.; Orduna-Malea, E.; Ayllón, J. M.; Delgado López-Cózar, E. (2016). A two-sided academic landscape: snapshot of highly-cited documents in Google Scholar (1950-2013). Revista Española de Documentacion Cientifica, 39(4). http://doi.org/10.3989/ redc.2016.4.1405

Martín-Martín, A.; Orduna-Malea, E.; Thelwall, M,; Delgado López-Cózar, E. (2018). Google Scholar, Web of Science, and Scopus: A systematic comparison of citations in 252 subject categories. Journal of Informetrics, 12(4), 1160-1177. https://doi.org/10.1016/]. JOI.2018.09.002

Masip, P. M. (2011). Efecto ANECA: producción española en comunicación en el Social Science Citation Index. Anuario ThinkEPI, 2011, v. 5, 206-210. https://recyt.fecyt.es/index.php/ThinkEPI/article/ view/30505/16061

Ministerio de Economía y Competitividad (2012). Estrategia española de Ciencias y Tecnología y de Innovación. Madrid: Ministerio de Economía y Competitividad. Disponible en: https://bit.ly/2UzAXEV. 
Orduna-Malea, E.: Delgado López-Cózar, E. (2014). Google Scholar Metrics evolution: an analysis according to Languages. Scientometrics, 98 (3), 2353-2367. https://doi.org/10.1007/s11192-013-1164-8

Orduna-Malea, E.; Martín-Martín, A.; Delgado López-Cózar, E. (2017). Google Scholar as a source for scholarly evaluation: A bibliographic review of database errors. Revista Espanola de Documentacion Cientifica, 40(4), 1-33. http://doi.org/10.3989/redc.2017.4.1500

Piñeiro-Otero, T. (2016). La radio en la investigación comunicativa en España: Una línea minoritaria para un medio mayoritario. Signo y Pensamiento, 35(69), 3048. https://doi.org/10.11144/Javeriana.syp35-69.rice

Piñeiro-Otero, T. (2018). Problemática universalización de los indicadores de evaluación científica. Crítica desde la investigación en comunicación radiofónica. El Profesional de la Información, 27(2), 254. https://doi. org/10.3145/epi.2018.mar.04

Recalcati, M. (2016). La hora de clase. Por una erótica de la enseñanza. Barcelona: Anagrama.

Repiso, R,; Torres-Salinas, D.; Aguaded, I. (2019). La gestión de revistas: mérito de transferencia universal. Justo y necesario. Anuario ThinkEPI, v. 13. https://doi. org/10.3145/thinkepi.2019.e13e03

Repiso, R.; Orduna-Malea, E.; Aguaded, I. (2019). Revistas científicas editadas por universidades en Web of Science: características y contribución a la marca universidad. El profesional de la información, v. 28, n. 4, e280405. https://doi.org/10.3145/epi.2019.jul.05

Rodríguez-Serrano, A.; Palao-Errando, J. A; Marzal-Felici, J. (2019). Los estudios fílmicos en el contexto de las ciencias sociales: un análisis de autores, objetos y metodologías en las revistas de impacto españolas (2012-2017). BiD: textos universitarios de biblioteconomía y documentación, 43. https://dx.doi. org/10.1344/BiD2019.43.7
Saperas, E. (2016). Cuatro décadas de investigación comunicativa en España. Los procesos de institucionalización y de profesionalización de la Investigación (1971-2015). Disertaciones. Anuario Electrónico Estudios de Comunicación Social, 9(2), 27-45. https://doi. org/10.12804/disertaciones.09.02.2016.02

Soriano, J. (2008). El "efecto ANECA". En: I+C Investigar la Comunicación. Actas del Congreso Internacional Fundacional de la Asociación Española de Investigación de la Comunicación (AE-IC). Santiago de Compostela: Universidad de Santiago de Compostela. Disponible en: https://www.researchgate.net/publication/261780208_El_efecto_ANECA (21-08-2019).

Soriano, J. (2017). La política estatal de recompenses als investigadors en comunicació: una anàlisi crítica. Comunicació: Revista de Recerca i Anàlisi, 34(2), 33-51. http://dx.doi.org/10.2436/20.3008.01.159

Túñez-López, J. M.; Martínez-Solana, M. Y.; Valarezo-González, K. (2014). Análisis de productividad, impacto e índice h de la investigación en Comunicación a través de los perfiles personales en Google Académico. Revista Latina de Comunicación Sociali, 69, 684-709. https://doi.org/10.4185/RLCS-2014-1030

Tur-Viñes, V.; Núñez-Gómez, P. (2018). Spanish academic research groups in communication. Communication and Society, 31(4), 173-192. https://doi. org/10.15581/003.31.4.173-192

Zafra, R. (2017). El entusiasmo: Precariedad y trabajo creativo en la era digital. Barcelona: Anagrama.

Zunzunegui, S.; Zumalde, I. (2018). La era del saber fungible. En: Rodríguez Serrano, Aaron; Gil Soldevilla, Samuel (eds.), Investigar en la era neoliberal. Visiones críticas sobre la investigación en comunicación en España. Barcelona, Bellaterra, Castellón y Valencia: Universitat Pompeu Fabra, Universitat Autònoma de Barcelona, Universitat Jaume I y Universitat de València, Col.lecció Aldea Global, pp. 55-70. 